\title{
Nakedly singular counterpart of Schwarzschild's incompressible star. A barotropic continuity condition in the center
}

\section{Łukasz Bratek $^{1}$ (D) Joanna Jałocha ${ }^{1} \cdot$ Andrzej Woszczyna $^{1,2}$}

Received: 25 February 2019 / Accepted: 24 October 2019 / Published online: 6 November 2019 (c) The Author(s) 2019

\begin{abstract}
A static sphere of incompressible fluid with uniform proper energy density is considered as an example of exact star-like solution with weakened central regularity conditions characteristic of a nakedly singular spherical vaccuum solution. The solution is a singular counterpart of the Schwarzschild's interior solution. The initial condition in the center for general barotropic equations of state is established.
\end{abstract}

Keywords Naked singularities $\cdot$ Spherical symmetry $\cdot$ Stellar structure $\cdot$ Equilibrium states $\cdot$ Schwarzschild's interior solution

\section{Introduction}

Curvature singularities can be characterised in terms of scalars formed out of the Riemann tensor. A set of 16 independent real scalars is provided by CarminatiMcLenaghan invariants [1]. An arbitrary invariant of the curvature tensor can be expressed in terms of these scalars. For perfect fluid solutions at most 9 of the scalars are independent, depending on the Petrov type.

For static spherically symmetric perfect fluid solutions of Einstein equations [2] one can verify by direct calculation that only 3 of the curvature scalars are independent, for example $R_{a}^{a}, S^{a}{ }_{b} S^{b}{ }_{a}$ and $C^{a b}{ }_{c d} C^{c d}{ }_{a b}$ (here $R_{a b}$ is the Ricci tensor, $S_{a b}$ is the Plebański tensor and $C_{a b c d}$ is the Weyl tensor). Then all of the nonvanishing Carminati-McLenaghan invariants are functions of the three scalars. Up to constant factors, the three scalars are respectively: $(\rho(r)-3 p(r)),(p(r)+\rho(r))^{2}$ and $r^{-6}\left(M(r)+M_{g}-\frac{4}{3} \pi r^{3} \rho(r)\right)^{2}$, where $M(r)=4 \pi \int_{0}^{r} \tilde{r}^{2} \rho(\tilde{r}) \mathrm{d} \tilde{r}$ and $M_{g}$ is an

Łukasz Bratek

lukasz.bratek@pk.edu.pl

1 Institute of Physics, Faculty of Materials Engineering and Physics, Cracow University of Technology, ul. Podchorążych 1, PL-30084 Kraków, Poland

2 Copernicus Center for Interdisciplinary Studies, ul. Sławkowska 17, PL-31016 Kraków, Poland 
integration constant with the dimension of mass. The invariants $R_{a}^{a}, S^{a}{ }_{b} S^{b}{ }_{a}$ involve only the density $\rho(r)$ and pressure $p(r)$ and therefore are related to each other by the equation of state. In the investigated context there are thus only 2 functionally independent curvature scalars, one may chose $R^{a}{ }_{a}$ and $C^{a b}{ }_{c d} C^{c d}{ }_{a b}$. The conformal invariant $C^{a b}{ }_{c d} C^{c d}{ }_{a b}$ involves the Misner-Sharp mass function [3] which is defined for a time-dependent spherically symmetric system as a purely geometric quantity

$$
m(r, t)=\frac{\tilde{R}(r, t)}{2 G}\left(1-g^{a b} \partial_{a} \tilde{R}(r, t) \partial_{b} \tilde{R}(r, t)\right)
$$

with $\tilde{R}$ being the areal radius and $(t, r)$ the co-moving coordinates of the fluid. For the static solution of interest in this paper one has simply $\tilde{R}(r, t)=r$ and so $m(r, t)=M(r)+M_{g}$. Then, the Misner-Sharp mass function splits into two parts: $M(r)$ measuring the amount of continuous matter enclosed within the areal radius $r$, and $M_{g}$ being an independent constant parameter. For a constant density solution, the conformal curvature invariant $C^{a b}{ }_{c d} C^{c d}{ }_{a b}$ reduces to $\frac{48 G^{2} M_{g}{ }^{2}}{c^{4} r^{6}}$, formally coinciding with the result for Schwarzschild's black hole. When $M_{g} \neq 0$, this function of $r$ contributes as the leading term in the conformal invariant, making it to blow up in the limit $r \rightarrow 0$. In this sense, $M_{g}$ is the conformal curvature counterpart of mass independent of the matter content.

The regularity requirements for the Riemann and metric tensors [4] are usually imposed as supplementary conditions on the solutions for spherical gaseous clouds. In particular, the integration constant $M_{g}$ is assumed to vanish for star-like solutions regular at the center, unlike for the vacuum solutions with the central singularity. However, despite the assumed regularity, some of gravitational collapse solutions have been observed to asymptotically settle to nakedly singular equilibrium configurations [5-11]. To model similar final endstates, or other hypothetical nakedly singular objects as such, the regularity conditions might be relaxed by allowing for central singularities with $M_{g}<0$. The degree of irregularity of the curvature invariants are in this case the same as for the Schwarzschild black hole.

With the relaxed conditions, one is faced with new interesting possibilities that could be tested. Take for example numerical integration of the equation of structure of a bounded spherical cloud with the neutron star polytrope equation of state. The resulting ADM mass turns out to lie in the range exceeding the Oppenheimer-Volkoff limit [12]. In this context it would be instructive to consider a simpler exactly integrable problem. Related to this is also the nature of the initial condition in the center that should be imposed on solutions with general barotropic equation of state. As shown in the next section, the presence of the degree of freedom $M_{g}<0$ modifies the analytic properties of the Tolman-Oppenheimer-Volkoff equation [2,13] to such an extent that the pressure required to keep in equilibrium a positive density barotropic matter becomes negative for radii small enough, unless both the density and pressure vanish in the center. As is found later, with a given barotrope it may be impossible to obtain a solution satisfying these conditions (as it occurs for a polytrope $p=\kappa \rho^{\Gamma}$ with $\Gamma>1$ ). This imposes a limitation on the forms of barotropic matter with regular pressure profile at the center that could support stars against disintegration. 
The pressure profile of a conformally singular incompressible spherical cloud of uniform proper density is found in an analytical way in Sect. 3. For large radii the pressure behaves as expected for a star-like solution, decreasing to zero with growing distance from the center. However, as one goes in the opposite direction towards the singularity, the pressure, after attaining a maximum, gradually decreases to zero and then becomes negative, attaining a unique value in the center. This solution is a nakedly singular counterpart of the non-singular Schwarzschild's interior solution [14].

\section{The central boundary condition}

The TOV triad of the equilibrium equations for a conformally singular spherical body consisting of baroropic material reads:

$$
\begin{aligned}
\chi^{\prime}(x) & =-\frac{\left(-\mu+\mathcal{M}(x)+x^{3} \chi(x)\right)(\chi(x)+\omega[\chi(x)])}{x(x+2 \mu-2 \mathcal{M}(x))}, \quad \mu>0, \\
\mathcal{M}^{\prime}(x) & =x^{2} \omega[\chi(x)], \quad \mathcal{M}(0)=0, \\
f(\chi, \omega) & =0 .
\end{aligned}
$$

The equations involve three dimensionless functions $\chi, \omega$ and $\mathcal{M}$ of a dimensionless variable $x=r / r_{o}$ ( $r$ is the areal radius coordinate). The functions describe, respectively, the pressure profile: $p(r)=p_{o} \chi\left(r / r_{o}\right)$, the mass density profile: $\rho(r)=\rho_{o} \omega\left[\chi\left(r / r_{o}\right)\right]$, and the integrated mass profile: $\mathcal{M}\left(r / r_{o}\right)=\frac{4 \pi}{m_{o}} \int_{0}^{r} \tilde{r}^{2} \rho(\tilde{r}) \mathrm{d} \tilde{r}$, $\mathcal{M}(0)=0$. Functions $\omega$ and $\chi$ are not independent but related by some equation of state $f(\chi, \omega)=0$, that is, $f(\chi(x), \omega[\chi(x)]) \equiv 0$ for any $x$. The dimensional parameters $p_{o}, \rho_{o}, m_{o}$ and $r_{o}$ are set so as to eliminate all inessential constants from the equations. ${ }^{1}$ The only free parameter left, the $\mu$, measures in units of $m_{o}$ the negative mass parameter in the conformal curvature, hence the total Misner-Sharp mass function is $m_{o}\left(\mathcal{M}\left(r / r_{o}\right)-\mu\right)$ (it is assumed that $\left.\mu>0\right)$.

The standard form of the metric tensor of a static and spherically symmetric spacetime in which the equilibrium equation acquires the above form, implies that the term $x+2 \mu-2 \mathcal{M}(x)$ in the denominator of the expression for $\chi^{\prime}(x)$ should be everywhere positive for a star-like solution. In the neighbourhood of $x=0$, this will be possible for a bounded $\omega$ only with $\mu \geq 0$. On the contrary, with $\mu<0$, either the condition that the velocity four-vector of the fluid be everywhere time-like could not be satisfied, or the signature of the metric form would be inappropriate for a genuine spacetime, even though Eq. 2.1 could be formally the same. Moreover, the asymptotic flatness with positive total mass requires that $\mathcal{M}(\infty)-\mu>0$. As follows from the expression for $\chi^{\prime}(x)$ in Eq. 2.1, the pressure will be a decreasing function of $x$ (like for an ordinary star in the equilibrium) only for $x$ large enough. For lower $x, \chi^{\prime}(x)$ may become positive and the pressure attain a local maximum. Such a maximum has been observed for a numerical model studied in [12].

$\overline{{ }^{1} \text { In writing Eq. } 2.1 \text { we have assumed: } \frac{4 \pi G r_{o}^{2} p_{o}}{c^{4}}=1, \frac{\rho_{o} c^{2}}{p_{o}}=1 \text { and }} \frac{G m_{o}}{r_{o} c^{2}}=1$. 
In finding solutions, especially numerical ones in the $x=0$ vicinity, it may prove helpful the following

Theorem If the pressure $\chi(x)$ is continuous at $x=0$ and continuously differentiable for $x>0$ (as expected for a star consisting of ordinary matter) then $\lim _{x \rightarrow 0}(\chi(x)+\omega[\chi(x)])=0$ for $\mu>0$ (implying that either $\chi(0)=0=\omega(0)$, or $\chi(0)=-\omega(0)$ for $\omega(0) \neq 0)$.

Proof Making use of the mean value theorem applied to function $\chi(x)$, it follows with the use of Eq. 2.1 that

$$
\chi(x)-\chi(0)=\frac{\chi\left(x \eta_{x}\right)+\omega\left[\chi\left(x \eta_{x}\right)\right]}{\eta_{x}} \cdot \frac{\mu-\mathcal{M}\left(x \eta_{x}\right)-\eta_{x}^{3} x^{3} \chi\left(x \eta_{x}\right)}{2 \mu+x \eta_{x}-2 \mathcal{M}\left(x \eta_{x}\right)},
$$

where $\eta_{x}$ is an $x$-dependent number such that $0<\eta_{x}<1$ and $\eta_{x} \rightarrow 0$ as $x \rightarrow 0$. With bounded $\chi$ and $\omega$, both $\mathcal{M}(x)$ and $x^{3} \chi(x)$ tend to 0 . As so, these functions can be made arbitrarily small numbers compared with $\mu$ for any $x$ small enough. Now, taking the limit in the above formula, one obtains

$$
0=\lim _{x \rightarrow 0} \frac{\chi\left(x \eta_{x}\right)+\omega\left[\chi\left(x \eta_{x}\right)\right]}{2 \eta_{x}} \Rightarrow \chi(0)+\omega[\chi(0)]=0
$$

which ends the proof. With $\mu=0$ the above implication would not follow, because then $\lim _{x \rightarrow 0} \frac{0-\mathcal{M}(x)-x^{3} \chi(x)}{0+x-2 \mathcal{M}(x)}=\lim _{x \rightarrow 0} \frac{-\mathcal{M}^{\prime}(x)-3 x^{2} \chi(x)-x^{3} \chi^{\prime}(x)}{1-2 \mathcal{M}^{\prime}(x)}=0$ if only $x^{3} \chi^{\prime}(x) \rightarrow 0$, with the same assumptions as before.

In accordance with the theorem, for barotropes with non-negative $\omega$ and with $\chi$ continuous at $x=0$, one infers that $\chi(0)=-\omega[\chi(0)]<0$ or $\chi(0)=0=\omega[0]$. For instance, the pressure becomes negative in a neighbourhood of the center for the exact solution presented in Sect. 3. But negative pressure is impossible for matter described by a polytropic equation of state for which both the density and pressure are required non-negative. The only possibility for such a polytrope is that either $\chi(0)=0$ as follows from the above theorem, or the reservations of the theorem are not met for a given solution. On that account, assume that in the limit of low pressure, matter can be described by a polytrope $\omega(\chi)=a \chi^{\gamma}$ with $\gamma>0$ (usually it is expected that $\omega(\chi) \sim a \chi^{n /(n+1)}, n>0$ for realistic polytropes). With $0<\gamma<1$, like for ordinary matter, $\chi+a \chi^{\gamma} \sim a \chi^{\gamma}$ as $\chi \rightarrow 0$, then, upon integration of the approximated equation $\chi^{\prime}(x) \approx(\chi(x)+\omega[\chi(x)]) /(2 x)$, one gets $\chi^{1-\gamma} \sim a(1-\gamma) \ln (\sqrt{x})+c-$ a contradiction with the assumed initial condition $\chi(0)=0$ (again, in writing the approximated equation one makes use of the fact that $\mathcal{M}$ and $x^{3} \chi$ are negligible compared to $\mu$ for finite $\chi$ and $x$ small enough). Hence, a solution with a polytropic equation of state and finite pressure would be possible at $x=0$ only for $\gamma \geq 1$, in which case $\chi \sim c \sqrt{x}$ as $x \rightarrow 0$. For a polytrope with $0<\gamma<1$ a solution with vanishing initial pressure would have to start at some $x=x_{0}>0$ and behave like $\chi \sim c\left(x-x_{o}\right)^{\frac{1}{1-\gamma}}$ as $x \rightarrow x_{o}^{+}$. A similar line of reasoning for the particular case of polytropic equations of state, showing that $\chi(0)=0$ for $\gamma \geq 1$ and that finite positive 
$\chi(0)$ is incompatible with $0<\gamma<1$, was presented by Oppenheimer and Volkoff

[2]. The theorem as given above for barotropic equations of state is more general.

\section{Interior solutions}

This section considers static equilibrium of a conformally singular in the centre spherical star consisting of incompressible fluid with constant proper mass density. Let $a$ be the areal radius of the star, $M$ the total mass of the regular mass distribution of the star, and $-M_{o}\left(M_{o}>0\right)$ the negative mass parameter in the conformal curvature invariant. The three quantities determine two independent dimensionless parameters $\alpha$ and $\mu$ of the model

$$
\alpha:=\frac{a c^{2}}{2 G M}>1-\mu, \quad \mu:=\frac{M_{o}}{M} \in(0,1)
$$

(the condition $\alpha>1-\mu$ will become clear later). In this model the dimensionless pressure profile $\chi$ and the uniform density profile $\omega$ are defined by

$$
p(r)=p_{o} \cdot \chi(r / a) \quad \text { and } \quad \frac{\rho(r)}{\rho_{o}}=\omega(\chi(x)) \equiv 1, \quad \text { with } \quad \frac{p_{o}}{c^{2}}=\rho_{o}=\frac{M}{\frac{4}{3} \pi a^{3}} .
$$

The areal radius $a$ can be chosen as the unit of length: $r_{o}=a$. Accordingly, $x=r / a$ is the dimensionless radial variable and the interval $0<x<1$ describes the star interior. The Misner-Sharp mass function is $\mathcal{M}(x)-\mu=x^{3}-\mu$ for $0<x<1$ (it can be verified that Komar mass will be different and involve also $\chi(x)$, however, both masses will overlap at $x=1$ and be equal to $1-\mu$ ). In these units, for the general form of the line element

$$
\mathrm{d} s^{2}=-e^{2 \Psi(x)} c^{2} \mathrm{~d} t^{2}+a^{2}\left(\frac{\mathrm{d} x^{2}}{1-\frac{\mathcal{M}(x)-\mu}{\alpha x}}+x^{2}\left(\mathrm{~d} \theta^{2}+\sin ^{2} \theta \mathrm{d} \phi^{2}\right)\right),
$$

the equilibrium equation to be satisfied by the pressure profile $\chi(x)$ acquires the following form

$$
\chi^{\prime}(x)=-\frac{(1+\chi(x))\left(x^{3}(1+3 \chi(x))-\mu\right)}{2 x\left(-x^{3}+x \alpha+\mu\right)}, \quad \begin{array}{r}
0<x<1, \\
\chi(1)=0 .
\end{array}
$$

Once the equation is solved, the other metric function satisfying the equation $\Psi^{\prime}(x)=$ $-\frac{\chi^{\prime}(x)}{1+\chi(x)}$ is easily found

$$
e^{2 \Psi(x)}=\frac{C}{(1+\chi(x))^{2}}, \quad C=1+\frac{\mu-1}{\alpha},
$$

where the integration constant $C$ is set by the requirement that for $x>1$ the resulting metric must be extended by the external Schwarzschild's vacuum metric with total mass $1-\mu>0$. The conformal curvature scalar for this solution is 


$$
C^{a b}{ }_{c d} C^{c d}{ }_{a b}=\frac{48 G^{2} M^{2}}{c^{4} r^{6}} \cdot\left\{\begin{array}{c}
\mu^{2}, 0<r<a \\
(1-\mu)^{2}, \quad r>a
\end{array}\right.
$$

and diverges at the center. The invariant is discontinuous on the star boundary $r=a$ unless $\mu=1 / 2$.

\subsection{Nonsingular interior solution $(\mu=0)$}

The solution for $\mu=0$ was found by Schwarzschild in 1916 [14]. It is known as Schwarzschild's interior solution. In our notation the solution reads:

$$
\left.\chi(x)\right|_{\mu=0}=\frac{\sqrt{\alpha-x^{2}}-\sqrt{\alpha-1}}{3 \sqrt{\alpha-1}-\sqrt{\alpha-x^{2}}}, \quad \alpha>\frac{9}{8} .
$$

The above condition for $\alpha$ assures that the pressure is finite (and everywhere nonnegative). Although there is a region of negative pressure for $\alpha<9 / 8$, such solutions are of interest today. As noticed by Mazur and Mottola [15], the divergence in pressure at $x=3 \sqrt{1-\frac{8}{9} \alpha}$ is integrable and the solution with the distinguished value $\alpha=1$ can be reinterpreted as a gravitational condensate star with constant negative pressure $\chi=-\omega=-1$ throughout the star and some residual transverse stresses at the boundary of the star.

\subsection{Nakedly singular interior solution $(\mu>0)$}

The Schwarzschild's interior solution is qualitatively changed in the $x=0$ vicinity if $\mu>0$. Then, for $\chi$ continuous at $x=0$, there is a $\delta>0$ such that $\chi^{\prime}(x) \sim$ $(1+\chi(x)) /(2 x)$ for $x<\delta$, hence $\chi(x) \sim-1+A \sqrt{x}$, proving that $\chi(0)=-1$. This is consistent with the theorem given in Sect. 2. Note that $\chi(0)=-1$, independently of the integration constant $A$ the value of which is controlled by the other part of the solution for larger $x$. Namely, $A$ in the asymptotic form of $\chi$ at $x=0$ is determined by the value $\chi=0$ at the boundary $x=1$.

A substitution

$$
\chi(x)=\frac{2}{3 u(x)}-1
$$

transforms the non-linear equation 3.1 for $\chi$, to a linear equation for $u$, with rational functions as coefficients:

$$
u^{\prime}(x)+\frac{2 x^{3}+\mu}{2 x\left(-x^{3}+x \alpha+\mu\right)} u(x)=\frac{x^{2}}{-x^{3}+x \alpha+\mu} .
$$

Before proceeding further, the roots of the denominators in the linear equation have to be examined. In this respect it is convenient to introduce the following combination of numbers $\alpha$ and $\mu$ : 


$$
v=\sqrt{\frac{27 \mu^{2}}{4 \alpha^{3}}}, \quad \text { or equivalently } \quad v=\frac{M_{o}}{2 M}\left(\frac{6 G M}{a c^{2}}\right)^{3 / 2} .
$$

A new dimensionless parameter $v$ can be regarded as a discriminant of the trinomial $w(x)=-x^{3}+\alpha x+\mu$ characteristic of the investigated problem. For $v<1$ the roots of the qubic equation $w(x)=0$ are all real and read

$$
x_{k}=2 \sqrt{\frac{\alpha}{3}} \cos \left(\frac{2 k \pi}{3}+\frac{1}{3} \operatorname{Arccos} v\right), \quad k=0,1,2, \quad v<1
$$

Then $x_{0}>\sqrt{\alpha}, x_{1}<0$ and $x_{2}<0$. For $v>1$ only one root is real and reads $2 \sqrt{\frac{\alpha}{3}} \cosh \left(\frac{1}{3} \operatorname{Arcosh} v\right)>\sqrt{\alpha}$. For physical reasons, it is important that the roots be located exterior to the stellar interior $0<x \leq 1$. This will be the case if one safely assumes independently of $\mu$ that $\alpha>1$, that is, when the coordinate radius of the star is greater than its Schwarzschild's radius: $a>\frac{2 G M}{c^{2}}$. In fact, with a given $\mu$ the $\alpha$ could be assumed lower: $\alpha>1-\mu$, then $w(x)>-x^{3}+(1-\mu) x+\mu>0$ for any $0<x<1$ and $0<\mu<1$.

Coming back to Eq. 3.3, with the reservations for the roots made, the linear equation may be recast into the equivalent form

$$
\frac{\partial}{\partial x} \frac{\sqrt{x} u(x)}{\sqrt{-x^{3}+x \alpha+\mu}}=\frac{x^{5 / 2}}{\left(-x^{3}+x \alpha+\mu\right)^{3 / 2}},
$$

hence the general solution involves an elliptic integral and reads

$$
u(x)=\sqrt{\frac{-x^{3}+x \alpha+\mu}{x}} \int \frac{x^{\frac{5}{2}}}{\left(-x^{3}+\alpha x+\mu\right)^{\frac{3}{2}}} d x .
$$

In terms of a new integration variable

$$
s(x)=-\frac{1}{2}-\frac{v}{x} \sqrt{\frac{\alpha}{3}}
$$

the solution can be expressed in a form with standardized elliptic integral

$$
\frac{u(x)}{\sqrt{\frac{-x^{3}+x \alpha+\mu}{\alpha x}}}=C+\frac{v^{2} \sqrt{6}}{4} \mathcal{I}(x), \quad \mathcal{I}(x)=\int_{-\infty}^{s(x)} \frac{\mathrm{d} \tilde{s}}{\sqrt{\left(s_{3}-\tilde{s}\right)^{3}\left(s_{2}-\tilde{s}\right)^{3}\left(s_{1}-\tilde{s}\right)^{3}}} .
$$

Parameters $s_{n}$ in the integrand are given by

$$
s_{n}=\cos \left(\frac{2}{3} \operatorname{Arcsin} v+\frac{2 n \pi}{3}\right), \quad n=1,2,3 .
$$




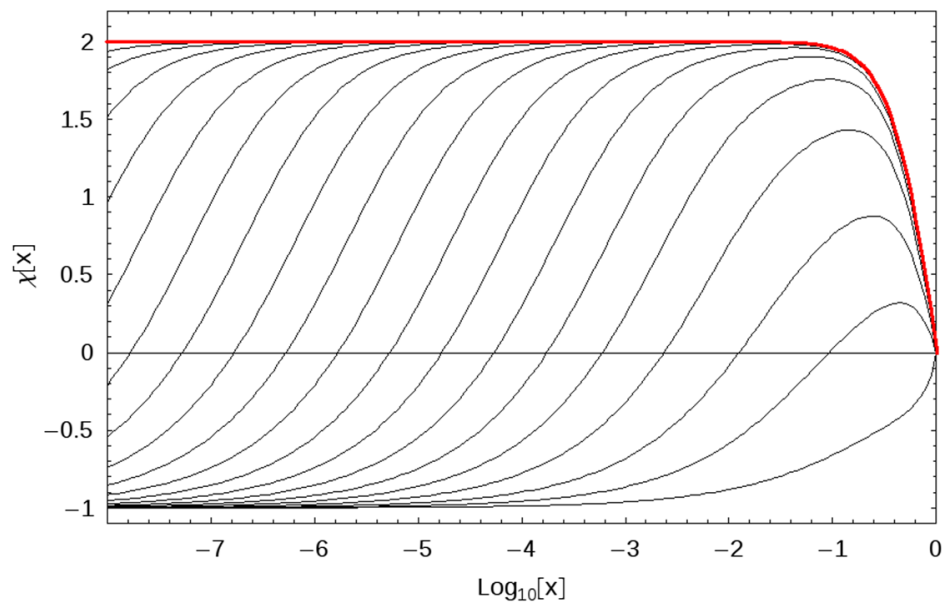

Fig. 1 The exact pressure profile $\chi(x)$ for the constant density ball with central conformal singularity, shown for various $\mu>0$ [thin lines]. For $x>0$, as $\mu \rightarrow 0$, the profiles tend (non-uniformly) to the $\mu=0$ regular Schwarzschild's interior solution profile [thick line], while $\chi(0)$ remains fixed by -1 for any $\mu>0$

They are the roots of the qubic form $-s^{3}+\frac{3}{4} s+\frac{1-2 v^{2}}{4} \equiv\left(s_{3}-s\right)\left(s_{2}-s\right)\left(s_{1}-s\right)$, $\left(s_{3}>s_{2}>s_{1}\right)$. They correspond to the roots of the previous qubic form $w(x)$ : $x_{k}=-\frac{2 v}{1+2 s_{k+1}} \sqrt{\frac{\alpha}{3}}, k=0,1,2$. The integration constant $C$ is set by the condition $\chi(1)=0(u(1)=2 / 3)$ on the surface of the star. Hence, the final solution for $0<x<1$ reads

$$
\chi(x)=\frac{\sqrt{\frac{x(-1+\alpha+\mu)}{-x^{3}+x \alpha+\mu}}}{1-\frac{81 \sqrt{6}}{32} \frac{\mu^{2}}{\alpha^{3}} \sqrt{\frac{-1+\alpha+\mu}{\alpha}}(\mathcal{I}(1)-\mathcal{I}(x))}-1, \quad \alpha>1-\mu .
$$

The explicit form of the solution is very intricate; for clarity of the presentation the integral $\mathcal{I}(x)$ is given in its entirety in "Appendix". To get some idea of how the pressure profile looks like anyway, it is presented in the graphical form in Fig.1. Outside the center $x>0$, the pressure tends point-wise in the limit $\mu \rightarrow 0$ to the interior Schwarzschild's solution Eq. 3.2 with $\mu=0$. Since for $\mu>0$ the pressure at $x=0$ is fixed by a negative number $\chi(0)=-1$ independent of $\mu$, this limit is non-uniform.

Open Access This article is distributed under the terms of the Creative Commons Attribution 4.0 International License (http://creativecommons.org/licenses/by/4.0/), which permits unrestricted use, distribution, and reproduction in any medium, provided you give appropriate credit to the original author(s) and the source, provide a link to the Creative Commons license, and indicate if changes were made. 


\section{Appendix}

Let $s_{1}, s_{2}$ and $s_{3}$ be defined as in Eq. 3.5, the function $s(x)$ as in Eq. 3.4. The definite improper integral $\mathcal{I}(x)$ present in the expression for the pressure profile in Eq. 3.6, has standard form of an elliptic integral that can be found in tables of integrals, and it reads (see for example integral 3.136.1 page 263 in [16]):

$$
\begin{aligned}
\mathcal{I}(x):= & \int_{-\infty}^{s(x)} \frac{\mathrm{d} \tilde{s}}{\sqrt{\left(s_{3}-\tilde{s}\right)^{3}\left(s_{2}-\tilde{s}\right)^{3}\left(s_{1}-\tilde{s}\right)^{3}}} \\
= & \frac{2}{\left(s_{3}-s_{2}\right)^{2}\left(s_{2}-s_{1}\right)^{2} \sqrt{\left(s_{3}-s_{1}\right)^{3}}} \times\left\{\left(s_{2}-s_{1}\right)\left(s_{3}+s_{2}-2 s_{1}\right) F(\varphi(x), \kappa)\right. \\
& \left.-2\left(s_{1}{ }^{2}+s_{3}^{2}+s_{2}{ }^{2}-s_{3} s_{2}-s_{3} s_{1}-s_{2} s_{1}\right) E(\varphi(x), \kappa)\right\} \\
& +\frac{2\left[s_{1}\left(s_{3}-s_{1}\right)+s_{2}\left(s_{3}-s_{2}\right)-s(x)\left(2 s_{3}-s_{1}-s_{2}\right)\right]}{\left(s_{3}-s_{2}\right)\left(s_{3}-s_{1}\right)\left(s_{2}-s_{1}\right)^{2} \sqrt{\left(s_{3}-s(x)\right)\left(s_{2}-s(x)\right)\left(s_{1}-s(x)\right)}},
\end{aligned}
$$

where

$$
\varphi(x)=\operatorname{Arcsin} \sqrt{\frac{s_{3}-s_{1}}{s_{3}-s(x)}}, \quad \kappa=\sqrt{\frac{s_{3}-s_{2}}{s_{3}-s_{1}}} .
$$

Here, $F$ and $E$ are the incomplete elliptic integrals of the first and second kind, as defined in the same tables of integrals [16]:

$$
F(\phi, k)=\int_{0}^{\phi} \frac{\mathrm{d} \theta}{\sqrt{1-k^{2} \sin ^{2} \theta}}, \quad E(\phi, k)=\int_{0}^{\phi} \sqrt{1-k^{2} \sin ^{2} \theta} \mathrm{d} \theta
$$

The form of the expression on the right hand side of the equality sign in the above definition of $\mathcal{I}(x)$ assumes that $s_{3}>s_{2}>s_{1}>s(x)$.

\section{References}

1. Carminati, J., McLenaghan, R.G.: Algebraic invariants of the Riemann tensor in a four-dimensional Lorentzian space. J. Math. Phys. 32, 3135-3140 (1991)

2. Oppenheimer, J.R., Volkoff, G.M.: On massive neutron cores. Phys. Rev. 55, 374-381 (1939)

3. Misner, C.W., Sharp, D.H.: Relativistic equations for adiabatic, spherically symmetric gravitational collapse. Phys. Rev. 136, B571-B576 (1964)

4. Wald, R.M.: General Relativity. Chicago Univ. Pr, Chicago (1984)

5. Joshi, Pankaj S. (ed.): Gravitational Collapse and Spacetime Singularities. Cambridge Monographs on Mathematical Physics. Cambridge University Press, Cambridge (2012)

6. Joshi, P.S., Malafarina, D., Narayan, R.: Equilibrium configurations from gravitational collapse. Class. Quantum Gravity 28(23), 235018 (2011)

7. Malafarina, D., Joshi, P.S.: Gravitational collapse with tangential pressure. Int. J. Mod. Phys. D 20, 463-495 (2011) 
8. Joshi, P.S., Malafarina, D.: Recent developments in gravitational collapse and spacetime singularities. Int. J. Mod. Phys. D 20, 2641-2729 (2011)

9. Joshi, P.S., Malafarina, D., Saraykar, R.V.: Genericity aspects in gravitational collapse to black holes and naked singularities. Int. J. Mod. Phys. D 21(08), 1250066 (2012)

10. Joshi, P.S., Malafarina, D.: Instability of black hole formation under small pressure perturbations. Gen. Relativ. Gravit. 45(2), 305-317 (2013)

11. Joshi, P.S., Malafarina, D., Narayan, R.: Distinguishing black holes from naked singularities through their accretion disc properties. Class. Quantum Gravity 31(1), 015002 (2013)

12. Woszczyna, A., Kutschera, M., Kubis, S., Czaja, W., Plaszczyk, P., Golda, Z.A.: Nakedly singular non-vacuum gravitating equilibrium states. Gen. Relativ. Gravit. 48(1), 5 (2015)

13. Tolman, R.C.: Static solutions of Einstein's field equations for spheres of fluid. Phys. Rev. 55, 364-373 (1939)

14. Schwarzschild, K.: Über das Gravitationsfeld einer Kugel aus inkompressibler Flüssigkeit nach der Einsteinschen Theorie. Preussische Akademie der Wissenschaften, Sitzungsberichte, 1916 (Erster Halbband), Berlin (1916)

15. Mazur, P.O., Mottola, E.: Surface tension and negative pressure interior of a non-singular black hole. Class. Quantum Gravity 32(21), 215024 (2015)

16. Gradshteyn, I.S., Ryzhik, I.M.: Table of Integrals, Series, and Products, eighth edn. Elsevier, Amsterdam (2015)

Publisher's Note Springer Nature remains neutral with regard to jurisdictional claims in published maps and institutional affiliations. 